\title{
Les possibles corporels : support biologique, determinations sociales
}

Bodily possibilities : biological basics and social determinants

\section{Manuel Schotté}

\section{(2) OpenEdition \\ 1 Journals}

Édition électronique

URL : http://journals.openedition.org/ress/3520

DOI : $10.4000 /$ ress.3520

ISBN : $1663-4446$

ISSN : $1663-4446$

Éditeur

Librairie Droz

Édition imprimée

Date de publication : 15 mai 2016

Pagination : 201-220

ISSN : 0048-8046

Référence électronique

Manuel Schotté, «Les possibles corporels : support biologique, determinations sociales ", Revue européenne des sciences sociales [En ligne], 54-1 | 2016, mis en ligne le 15 mai 2019, consulté le 03 janvier 2020. URL : http://journals.openedition.org/ress/3520 ; DOI : 10.4000/ress.3520 


\title{
LES POSSIBLES CORPORELS: SUPPORT BIOLOGIQUE, DÉTERMINATIONS SOCIALES
}

\author{
MANUEL SCHOTTÉ \\ Université de Lille - CERAPS \\ manuel.schotte@univ-lille2.fr
}

Résumé. L'étude des coureurs à pied de compétition s'avère particulièrement propice pour analyser les liens entre le social et le biologique, en ce qu'elle conduit à réfléchir à ce qui détermine les capacités corporelles d'un individu. Dans cette optique, trois pistes sont explorées: la première s'intéresse à la manière par laquelle le social détermine les corps dans leur matérialité même; la deuxième met l'accent sur le façonnement social des catégories de perception et montre que la fatigue ressentie par un athlète s'inscrit à l'interface entre des manifestations physiologiques et un encodage symbolique de ces dernières; la troisième fait ressortir que, loin d'être réductibles à la mise en jeu de capacités physiques «pures», la performance procède d'une interaction qui détermine ce dont un coureur est capable. Considérés dans leur ensemble, ces trois développements visent à rendre compte de ce que le social fait à des potentialités physiologiques dont il n'y a pas lieu de penser qu'elles puissent être, à elles seules, au principe de différences interindividuelles en matière de possibles corporels.

Mots-clés: biologie, constructivisme, corps, déterminations sociales.

\begin{abstract}
The study of competitive runners proves particularly rich for analyzing connections between the social and the biological, as it leads us to consider what determines an individual's physical capacities. Accordingly, this article explores this question from three angles: the first looks at how the social determines the very materiality of the body; the second emphasizes how categories of perception are socially shaped, and shows that the fatigue athletes feel is situated at the interface of physiological manifestation and its symbolic coding; the third reveals that performance, far from boiling down to the expression of "pure" physical capacities, arises instead from an interaction that determines what a runner can accomplish. Taken together, these three aspects illustrate the influence of social facts on physiological potential, which leaves no reason to think that it could be the sole basis of interindividual differences in what the body may achieve.
\end{abstract}

Keywords: biology, body, constructivism, social determinants. 
Biologistes et sociologues se comportent comme si les aspects biologiques et sociaux des êtres humains étaient aussi rigoureusement séparés et indépendants les uns des autres que revendiquent de l'être leurs professions respectives, et en tout état de cause les départements universitaires de biologie et de sociologie [...] en réalité, les processus biologiques et sociaux dépendent les uns des autres.

(Elias, 2015 [1991], p. 54)

La performance en course à pied est très largement pensée au travers de catégories biologiques : n'importe quel manuel d'entraînement s'ouvre sur des considérations d'ordre physiologique et l'ensemble des conseils de préparation est envisagé suivant des grilles d'interprétation relevant de ce registre. Cette domination du prisme biologique, qui a sans doute beaucoup à voir avec le fait que ce sport est souvent perçu comme le plus naturel qui soit, conduit à une mainmise des chercheurs appartenant à ce domaine dans la production de connaissances sur cette pratique.

Une telle situation ne poserait pas de problème, si elle ne s'accompagnait pas d'une tendance au biologisme, c'est-à-dire de la tentation de faire dériver l'ensemble de ce qui se joue en course de lois biologiques. Suivant cette lecture, ce dont un athlète est capable serait réductible à des propriétés physiologiques : la performance se résumerait à une série d'équations calculant la consommation maximale d'oxygène (VO2 $\mathrm{Max}^{1}$ ), l'économie de course, le seuil anaérobie, etc. Il ne s'agit pas ici de contester l'intérêt, évident, des travaux ayant mis au jour ces différentes dimensions mais de critiquer leur prétention à un monopole en matière d'explication de ce qui fonde la performance et leur propension à naturaliser ce qui la sous-tend.

On pourrait être tenté, face à un tel impérialisme, de mobiliser une réponse symétrique venant à considérer que, loin d'être biologique, la performance ne relèverait que du social. Un tel souci d'affirmation disciplinaire aurait alors toutes les chances de sombrer dans une forme de sociologisme aussi funeste sur le plan de la connaissance que ce dont il prétend se démarquer: comment peut-on en effet nier que la performance sportive ait une assise biologique? Il convient donc

I Concept physiologique qui a donné son nom, c'est significatif, à l'une des deux principales revues françaises de course à pied: VO2 Magazine. 
d'éviter de penser en termes d'alternatives exclusives, et de mettre à distance les propositions qui raisonnent en termes de «ou bien, ou bien» (Elias, 2015 [199I]). Il ne s'agit pas pour autant de plaider pour un arbitrage mou consistant à affirmer par exemple que la performance en course à pied relève à $50 \%$ du biologique et à $50 \%$ du social. La question n'est effectivement pas de trancher la question de la nature des choses². Mais, s'il faut rejeter le principe d'un découpage ontologique des objets (qui amène à considérer que telle entité relève du social et telle autre du biologique, ou des deux dans telle et telle proportions), il est utile de dissocier sur un plan analytique ce qui est, dans la réalité, inextricable. Et la tâche du sociologue consiste alors à essayer de remonter aussi loin que possible dans la mise au jour de ce qui relève de l'emprise du social sur le biologique.

C'est ce que le présent article s'efforce de réaliser en partant du cas de la performance en course à pied. L'étude de ce sport s'avère particulièrement propice pour étudier les liens entre le social et le biologique, en ce qu'il conduit à réfléchir à ce qui délimite les possibles corporels d’un individu et ce qui les détermine. Il s'agit, dans ce cadre, de montrer que, là où la biologie prend principalement pour objet le fonctionnement du corps conçu comme un universel ${ }^{3}$, l'intérêt des sciences sociales réside dans leur capacité à mettre au jour ce qui conduit à différencier les humains entre eux (Lignier, 2015). Dans cette optique, trois pistes sont explorées: la première s'intéresse à la manière par laquelle le social détermine les corps dans leur matérialité même ; la deuxième met l'accent sur le façonnement social des catégories de perception des coureurs et montre que la fatigue ressentit par un coureur s'inscrit à

2 Dans la mesure où il est impossible de dissocier ce qui relève du social et du biologique. II n'y a, concernant les êtres humains, rien de biologique qui ne soit socialisé. Et, inversement, le biologique est le support nécessaire à la vie sociale. En ce sens, on peut dire que nous sommes 100\% biologiques et 100\% sociaux.

3 II ne s'agit pas de nier que la biologie puisse rendre compte de ce qui singularise les corps (comme le prouve la possibilité de reconnaître un individu par son ADN) mais de rappeler, d'abord, qu'elle vise à mettre au jour les lois de fonctionnement de l'organisme, et, ensuite, qu'elle ne saurait, dans son acception classique, rendre compte de ce qui différencie les comportements interindividuels, question qui relève du spectre des sciences sociales. D’ailleurs, comme le montre Maurizio Meloni (20l4a), les avancées récentes de la biologie vont elles-mêmes dans le sens d'une prise en compte accrue de l'importance du social dans la détermination des fonctions biologiques. 
l'interface entre des manifestations physiologiques et un encodage symbolique de ces dernières; la troisième fait ressortir que, loin d'être réductibles à la mise en jeu de capacités physiques «pures», la performance procède d'une interaction qui détermine ce dont un athlète est capable. Considérées dans leur ensemble, ces trois séquences - ordonnées et présentées de façon à permettre un affinement progressif du regard - visent à mettre en évidence que, si les possibles corporels s'appuient bien sur des potentialités biologiques, ils n’en sont pas moins déterminés socialement. La démonstration repose sur une enquête ethnographique auprès de coureurs marocains de tout niveau, depuis le débutant jusquau champion olympique ${ }^{4}$.

\section{LA TRANSFORMATION MATÉRIELLE DES CORPS}

La première manière d’envisager la façon dont le social détermine les possibles corporels réside dans la mise en évidence de la manière par laquelle il travaille les corps dans leur matérialité même. Cette perspective prend acte du fait que le corps présente «la propriété (biologique) d'être ouvert au monde, donc exposé au monde et, par là, susceptible d'être conditionné par le monde, façonné par les conditions matérielles et culturelles dans lesquelles il est placé dès l'origine» (Bourdieu, 1997, p. I6I). Même si c'est là une façon assez évidente d’aborder la question de l'emprise du social sur le biologique, force est de constater que cette perspective a été peu suivie en sciences sociales ${ }^{5}$. Même Marcel Mauss (1978 [1934]), qui insiste pourtant sur les variations culturelles en matière de techniques du corps, ne va pas jusqu'à montrer en quoi celles-ci s'accompagnent d'une transformation physique. S'il y a bien des allusions à l'inertie des apprentissages corporels ${ }^{6}$, cette dernière n'est pas référée à une éventuelle modification du corps en lui-même. Cet «oubli» est représentatif d'une tendance très générale. Alors même que la question du corps fait

4 Pour plus de détails sur cette enquête se reporter à Manuel Schotté (2012).

5 Pour une exception récente, voir Dieter Vandebroeck (20।5).

6 Notamment quand il évoque sa façon de nager qu'il décrit comme «stupide, mais je fais encore ce geste: je ne peux pas me débarrasser de ma technique» (Mauss, 1978 [1934], p. 367). 
désormais l'objet de nombreux travaux ${ }^{7}$, il est remarquable que la manière par laquelle il est matériellement affecté par le social demeure peu étudiée.

Il s’avère que, comme Loïc Wacquant (1995) l’a souligné, le sport constitue un très bon observatoire pour rendre compte de cette fabrique des corps. Son travail au sein d'un club de boxe de Chicago a mis en évidence à quel point le physique des boxeurs est transformé par leur pratique. Ce constat est également valable pour les coureurs à pied qui font, eux aussi, l'expérience personnelle de telles modifications biologiques, à l'instar de Brahim, dans l'extrait suivant :

Avant j'étais pas comme ça hein? [il montre ses joues, creusées]. C'est la préparation, tous les kilomètres. C'est comme ça que tu deviens sec. Celui qui n'a pas ça (les joues creuses) n’a pas bien entraîné.

Au-delà de ce ressenti subjectif et de l'attribution causale qui l'accompagne, de nombreux travaux de physiologie ont permis d'objectiver à quel point la pratique sportive intensive agit sur les corps et leur fonctionnement. Ces travaux mettent au jour que l'entraînement produit des transformations physiques précisément mesurables comme l'augmentation de la consommation maximale d'oxygène, la baisse de la fréquence cardiaque au repos, ou encore la transformation de la composition corporelle (diminution du pourcentage de masse grasse au profit de la masse musculaire), etc. C'est donc ici, de façon apparemment paradoxale, la physiologie qui offre les éléments les plus probants pour attester d'une marque du social sur les corps en montrant comment ces derniers sont affectés de façon différentielle suivant les conditions de préparation auquel on les soumet. Il suffit en effet de rappeler que l'entraînement est une action éminemment sociale - tant dans son orientation (qu'il vise la performance ou le bien-être, il répond toujours à des finalités socialement conditionnées) que dans sa forme (qu'il s'inscrive dans une relation pédagogique entre un entraîneur et un entraîné ou qu'il relève d'une préparation conduite de façon individualisée, il s’appuie toujours sur des savoirfaire collectifs, plus ou moins intériorisés) - pour voir quel intérêt ces travaux

7 Avec, au plan international, l'émergence d'une revue comme Body and Society qui apparaît en 1995. Cette revue, qui envisage avant tout le corps dans sa dimension symbolique et discursive, est représentative de cet oubli généralisé de la question de la fabrique matérielle des corps. 
de physiologie présentent pour les sciences sociales : en maniant expérimentalement les conditions de pratiques d'un individu pour mesurer leurs effets sur l'organisme, ils attestent de leur impact sur les corps.

C'est précisément en vue d'agir sur leur corps de façon à le rendre conforme aux exigences compétitives que les coureurs auprès desquels j’ai enquêté s'échinent, à raison de deux entraînements par jour, sept jours sur sept. Ils travaillent, pour reprendre les mots d'Ali, à «développer le cœur», «augmenter le souffle», et accroître «la force des jambes», et ce afin de devenir plus performants. Ils s'évertuent, au fil des kilomètres parcourus (de l'ordre de 150 par semaine, la plupart à haute intensité ${ }^{8}$ ), à «apprendre le train, faire rentrer la vitesse dans les jambes (d'un ton impératif et en pointant de son index ses cuisses comme s'il cherchait à l'y faire pénétrer) » comme le disait Brahim. L'ensemble de cette préparation vise à «adapter le corps à son usage » (Mauss, p. 385), qui est un usage sportif et qui conduit à une transformation du corps et de ses possibles. L'enquête ethnographique de longue durée m’a permis de constater - en particulier chez ceux qui ont débuté la pratique pendant les investigations - quelles transformations corporelles accompagnent ce très conséquent engagement dans la pratique : étirement des silhouettes, émaciement des visages, musculature qui s’affine et devient plus saillante, etc. C'est, à proprement parler, l'inscription d'une culture dans leur physiologie qui se donne ainsi à voir.

C'est parce qu'il offre la possibilité de repérer de telles modifications corporelles, et ce sur un temps court, que le sport constitue un très bon observatoire pour attester de l'empreinte du social sur la matérialité du corps99. Il permet de mettre en évidence la conditionnabilité du corps, conçue «comme capacité naturelle

8 Le kilométrage total traduit mal la charge d'entraînement, d'une part, parce que les kilomètres parcourus ne «valent» pas tous autant suivant la vitesse à laquelle ils sont effectués; d'autre part, parce que la préparation ne se limite pas aux séquences de course. S'y ajoutent les exercices en salle, musculation et étirements notamment.

9 C'est pourquoi on peut s'étonner que les travaux de sociologie du sport n'aient pas, en particulier en France, insisté davantage sur cette dimension qui peut paraître tellement évidente aux spécialistes de ce domaine qu'elle finit par ne pas être étudiée. II faudrait aussi s'interroger sur le poids des traditions et oppositions disciplinaires au sein des études sur le sport: 
d’acquérir des capacités non naturelles, arbitraires » (Bourdieu, 1997, p. 163). Si cette dimension appelle assurément des développements, notamment quant à la durabilité des transformations que rend possible cette malléabilité corporelle, sa seule mise au jour peut paraître suffisante à ce stade, tant elle est rarement prise en compte dans les travaux en sciences sociales.

\section{LE MODELAGE SENSORIEL}

Si l'évocation du façonnement social des corps est une dimension importante de la manière dont il faut articuler le social et le biologique, on ne peut pour autant se contenter de ce seul aspect. Comme l'a montré Wacquant (2000), la socialisation sportive ne se limite pas aux seules modifications corporelles strico sensu. Ces dernières s'accompagnent toujours d'un travail de mise en sens - dans toutes les acceptions du terme - de la pratique qui les sous-tend.

Les possibles d'un sportif ne peuvent en effet être envisagés comme résultant de son seul «potentiel biologique», fût-il considéré comme socialement construit. La pratique engage toujours du symbolique qui détermine aussi bien la signification investie que les catégories de perception mises en oeuvre durant l'effort. Les lignes qui suivent sont consacrées à ce second aspect en se focalisant sur la question de la fatigue que ressent un coureur en situation. Cet intérêt pour ce qu'on appelle fatigue se justifie dans la mesure où elle est souvent décrite comme le facteur limitant de la performance en course prolongée. Or, bien qu'elle soit le plus souvent renvoyée du côté de la biologie, elle n’est pas précisément définie par cette dernière, certains chercheurs relevant de ce domaine allant jusqu’à affirmer que les sensations de fatigue sont largement indépendantes des facteurs physiologiques (Noakes, 20I2). S’ouvre alors la possibilité de montrer que l'impression de fatigue s'inscrit, comme la sensation de douleur (Bendelow et Williams, 1995), à l’interface du social et du biologique.

tout se passe comme si le corps envisagé dans sa matérialité était conçu comme relevant du seul domaine des sciences de la vie. 
C'est ce que les lignes qui suivent s'efforcent de montrer, en insistant sur la façon par laquelle la pratique façonne les catégories de perception des coureurs et sur la manière dont ils interprètent ce qu'on appelle ordinairement la fatigue. Le cas du débutant est à cet égard particulièrement intéressant dans la mesure où, la pratique de la course conduisant à une perturbation physiologique (Wilmore et Costill, 1994), il éprouve des sensations qui lui était jusqu’alors inconnues (Bruant, 1992). La poursuite de son effort dépend de sa capacité à accepter ces dérèglements intérieurs et à supporter les sensations qui leur sont associées. Et comme pour les fumeurs de marijuana étudiés par Howard Becker (1985 [1963]), cela passe par un travail collectif d'accompagnement du novice pour lui faire accepter comme normales les altérations de son milieu interne. Potentiellement déstabilisantes, les manifestations physiologiques liées à l'effort sont normalisées par les pairs avec lesquels un athlète fait ses premiers pas, comme cette fois où Brahim et moi accompagnions les débuts athlétiques de son jeune frère Omar : alors que ce dernier se demandait, en milieu d'entraînement (un footing à allure modérée), s'il allait pouvoir nous suivre jusqu’à la fin, Brahim lui répondit sur un ton catégorique que : «là tu es juste un peu essoufflé. C’est rien, tu peux continuer. Pas de problème de nous suivre jusqu'à la fin».

En référant l'intensité de l'effort consenti par Omar à son degré d'essoufflement - repère perceptible pour un accompagnateur extérieur -, Brahim donne à son frère un moyen d'évaluer le niveau de difficulté de l'effort auquel il consent. Et, en catégorisant ce dernier comme relevant du soutenable, il le banalise. Brahim exprime d’ailleurs comment sa propre perception de la difficulté de l'effort a évolué :

- Au début, c'est dur. Tout le temps, tu crois que tu vas exploser... Le cœur, le souffle, les jambes. Toujours, c'est dur. T’as peur de pas tenir. Alors tu ralentis.

- Et aujourd'hui?

- Et ben aujourd'hui, tu sais que c'est normal quand c'est dur [rires]! Alors tu continues!» 
C'est à ce prix - celui de l'acceptation de la difficulté de la pratique comme horizon normal - que les coureurs acceptent d'entrer progressivement dans des zones d'effort de plus en plus inconfortables. Comme l'indique Brahim, le débutant a tendance à ralentir dès les premières difficultés. À l'inverse, le coureur expert sait «que c'est normal quand c'est dur» et a appris à ne pas céder et à ne pas se crisper, même quand l'effort est particulièrement intense, comme le témoigne cet athlète de rang international (son record personnel était de 3’33 sur I 500 mètres, ce qui le situait parmi les 20 meilleurs mondiaux) :

- Je t’ai vu la semaine dernière [il avait alors gagné une compétition en $3^{\prime} 38$ ]. Tu avais l'air facile.

- Ouais facile... mais pas facile en même temps! (rires)

- Pourquoi «facile» et «pas facile» en même temps?

- Bah «facile» parce que je savais que j'étais le plus costaud alors, j’ai gagné facile alors. Mais c'était dur quand même. Ah ouais, t’as la difficulté [et] la fatigue qui montent... Les bras, les jambes, tout.

- T’avais pas l'air de forcer pourtant!

- Ouais, je contrôlais mais c'était dur quand même hein!

La capacité à entrer dans des zones d'effort soutenu est indissociable d'une capacité à les supporter : là où le débutant est débordé par les effets de l'engagement intense, le coureur expérimenté est capable de soutenir l'expérience en les domestiquant. Au lieu de se laisser gagner par des émotions susceptibles d'entraver sa capacité à agir (Katz, 1999), il garde le contrôle de son investissement, même quand celui-ci est maximal. De sorte que le qualificatif de «difficile» se trouve accolé à des efforts de plus en plus soutenus: si le novice interprète les premiers signes d'altération de son milieu interne comme attestant d'un effort difficile, le coureur confirmé réserve l'usage de cet adjectif à des situations de mobilisation beaucoup plus grande. De sorte que ce n'est pas une plus grande endurance à la douleur qui les différencie mais une façon différente d'éprouver des stimuli physiologiques similaires (Boltanski, I97I). 
Même si cette dimension est essentielle, la socialisation des perceptions d'un coureur ne se limite pas à la question de la qualification - variable suivant le niveau d'expertise des athlètes - de l'intensité de la sensation. Le «modelage sensoriel» (Détrez, 2002, p. 97) de l'athlète conduit aussi à un changement dans l'identification même de ses signaux corporels. La comparaison avec les fumeurs de marijuana décrits par Howard Becker (1985 [1963]) vaut ici aussi : comme ces derniers, le coureur apprend, au contact de ses pairs et de son entraîneur, à discriminer plus finement ce qu'il ressent. À l'impression floue de fatigue qui caractérise le débutant quand il décrit ses sensations en course, se substitue progressivement une cartographie fine, et adossée à des registres d'effort, de ces dernières : les coureurs apprennent peu à peu à distinguer les efforts lactiques des efforts aérobies, à dissocier les sensations associées à chaque type d'exercice, etc. C'est ce genre de travail que permet Brahim dans l'extrait suivant, qui fait suite à un entraînement sur piste de son frère Omar. Brahim a supervisé cet entraînement en fixant le programme (une succession d'efforts courts à intensité maximale) et en tenant le chronomètre :

Bienvenue Omar! C'est ta première séance lactique... C'est dur hein? (Se tournant vers moi). T’as vu Manu, c'est la première fois qu'il sent le lactique. Il a pas l'habitude. (S’adressant de nouveau à son frère). C'est pas comme les footings, hein?

Le fait que l'ensemble des athlètes usent du même vocabulaire pour désigner leurs sensations ${ }^{10}$ ne doit pas conduire à inverser la perspective : ce qu'ils apprennent au contact de la communauté athlétique, ce n'est pas à mettre des mots sur des sensations qui préexisteraient et qui attendraient en somme d'être reconnues et désignées. À l'inverse, c'est le partage et les usages des mêmes notions qui les amènent à décrypter leur ressenti et à dissocier de la même façon les différents registres de fatigue auxquels les conduisent les différents types d'effort. Pour être perçue, la sensation doit faire l'objet d'une attention préalable. Et pour être «retenue» comme pertinente, elle doit être associée à un terme qui

I0 Le travail ethnographique montre qu'il existe toutefois de grandes différences internationales dans l'encodage symbolique de l'effort. Même si la «culture sportive» s'avère universelle - dans la mesure où les règles du jeu sont les mêmes partout dans le monde -, elle fait l'objet d'interprétations locales et nationales qui peuvent être très différentes (Besnier, 2012). 
permet sa désignation et sa transformation en catégorie de perception durable. Et comme l'indiquent les propos de Brahim, la grille d'interprétation qui prime dans cet encodage du monde interne est très largement nourrie de concepts issus de la biologie. Mais cet emprunt à la biologie est bien d'ordre social. Le fait que les catégories de perception des coureurs soient alimentées par ce filtre biologique ne signifie effectivement pas que leurs performances soient la stricte résultante de leur état physique (qui est, on l’a vu dans la première partie, socialement mise en forme). Elle s'inscrit au contraire au croisement de ce dernier et de la façon dont ils l'interprètent en situation car, loin de relever d'une «coloration » de manifestations physiologiques qui s'imposeraient d'elles-mêmes, l'encodage symbolique détermine la sensation et les possibles corporel ${ }^{11}$.

\section{LES POSSIBLES CORPORELS, PRODUITS D'UNE INTERACTION}

Si la manière d'envisager les relations entre le biologique et le social développée jusque ici demeure plus que jamais d’actualité, elle comporte le risque de cantonner le social à l'amont de l'action étudiée. Se limiter à ce travail - qui est, rappelons-le, déjà décisif et trop rarement mis en œuvre - de mise au jour de ce qui détermine la fabrique des corps et la façon de le percevoir peut conduire à perdre de vue ce qui se joue lorsque ces corps socialisés sont mis en jeu. Sauf à considérer que ce qui se passe en situation résulte de l'actualisation mécanique de propriétés de corps façonnés au préalable, il faut introduire dans l'analyse un second temps du social. Le social relève en effet d'un double mouvement: celui du temps long de la fabrication des dispositions et des capacités et celui du temps court de leur mise en jeu en situation. C'est à l'étude de ce second temps qu'est consacrée cette troisième partie de l'article, en montrant comment les possibles d'un athlète ne peuvent être réduits à la seule résultante de potentialités façonnées au préalable. Considérer que ces dernières ont une socio-genèse ne suffit pas. Il importe de montrer comment cette nature socialisée n’agit que dans sa

II On renvoie sur ce point au travail de Norbert Elias (1973 [1939]) qui montre comment les façons socialement conditionnées de percevoir le corps déterminent son usage et ses possibles via la genèse d'un auto-contrôle qui prend la forme d'un intériorisation de façons de faire (contrôle des sphincters, etc.). 
rencontre avec un contexte donné, ce qui confère une autonomie relative à la situation qui contribue à déterminer ce dont un athlète est capable.

Comme l'exprime Hicham, l'entraînement et la compétition sont deux moments très différents et on ne peut réduire ce qui se joue durant cette dernière au strict décalque de ce qui a été acquis lors de la phase de préparation :

J'comprends pas. Je suis bien-bien à l'entraînement. Et toujours la compétition ça va pas. J'comprends pas, vraiment. À l'entraînement, toujours je suis devant les autres [coureurs du quartier] et en course, ils sont devant! Younès il rigole. Toujours il dit que je suis le champion du monde de l'entraînement [rire désabusé]. Ah j'comprends pas. Je crois que ça vient de là [il montre son crâne avec son index].

Les apprentissages décrits plus haut ne sont pas suffisants pour conférer une maîtrise en situation lors d'une course. Résultant d'une interaction, le résultat compétitif ne saurait être conçu comme découlant d'une addition de capacités personnelles. De même que l'activité d'un musicien est fonction de celles des autres interprètes avec lesquels il joue (Schütz, I95 I Katz et Csordas, 2003), la performance en course à pied se construit dans et par une confrontation interindividuelle. C'est particulièrement vrai s'agissant du cross-country. Alors que les compétitions sur piste donnent l'occasion aux athlètes de s'étalonner via la connaissance de leurs temps de passage, les épreuves de cross interdisent cette possibilité : se déroulant sur des parcours à chaque fois différents, aux reliefs aléatoires et jamais métrés précisément, les concurrents n’y disposent d’aucun repère chronométrique pour se guider. Ils ne peuvent donc s'orienter qu'en fonction de leurs ressentis et des comportements de leurs adversaires, comme l'exprime ici Ali :

- C'est comme ça en cross... Tu peux pas courir avec le chrono... tu cours avec les autres, par rapport à les autres. Si ils accélèrent, tu accélères. Si ils ralentissent, tu ralentis. Ah ouais, c'est comme ça en cross. Tu cours pas par rapport à toi mais par rapport aux autres.

- Par rapport à tous les autres ? 
- Ah non, ceux qui sont comme moi [de même niveau]! Si je cours avec El Guerrouj, je vais pas le suivre, il est trop fort. Mais ceux qui sont comme moi... je regarde. Je regarde comment ils sont. Je me dis «alors lui, il est mort, je vais accélérer »... «lui il est facile, je vais rester derrière lui». Toujours tu fais comme ça en cross. Tu cours par rapport les [aux] autres.

Une course est le siège d'une intense activité de surveillance mutuelle. Chaque coureur ne cesse d'épier ses adversaires, d'évaluer leur degré de fatigue, d’ajuster son allure et de réagir en fonction de ce qu'ils font et de ce qu'il croit y déceler comme indices de leur valeur. Pris dans le feu croisé de ces vigilances réciproques, chaque concurrent essaie de trouver des preuves du niveau d'aisance ou de difficulté de ses opposants directs et par là de s'assurer qu'il est bien à sa place et que la suite des événements lui sera favorable. Dans cette quête, tout peut faire signe: une respiration bruyante, une grimace, une difficulté dans les relances, etc. (Collinson, 2008). Se sachant épiés, les coureurs tentent de dissimuler les manifestations d'effort et essaient de «paraître facile» pour mieux tromper et impressionner leurs adversaires. Si ces «jeux d'expression» (Goffman, 1970, p. 3) sont à ce point essentiels, c'est que chaque compétiteur court en fonction de ce qu'il perçoit des autres et de la façon dont il se situe par rapport à eux, comme le montre cet extrait d'entretien avec Brahim à l'issue d’une épreuve :

- À ce moment-là, j’ai fait une attaque et il a lâché.

- Pourquoi tu as attaqué à ce moment-là ?

- Bah, j’ai vu [qu’] il était mort.

- Comment tu le savais?

- Ben, il soufflait fort ou... Puis dans les virages, il avait du mal, sa foulée qui... Là, j’ai vu qu'il était mort, je sais qu'il va pas suivre si j’accélère. C'est comme ça toujours: toujours tu dois regarder les autres. Et quand tu vois qu'il y en a un qui est pas bien, tu peux faire l'attaque, il va lâcher. C'est comme ça qu'il faut faire.

- Pour toi c'est pareil ? Les autres ils doivent voir quand t'es cuit... 
- Non, parce que je le montre pas. C'est quand c'est dur qu'il faut faire attention. Tu restes comme ça [il se raidit et regarde impassible vers l'horizon], facile. Il faut être malin [rire]!

Le contrôle émotionnel (Hochschild, 1979) par lequel le coureur dissimule les effets de l'effort est inscrit dans un jeu de comparaison mutuelle. Bien que procédant d'un travail de soi sur soi, cette maîtrise est foncièrement collective dans son orientation: elle procède d'une visée extérieure qui conduit chaque coureur à donner la meilleure image de lui-même. Comme l'exprimait Ali plus haut, cette observation croisée est le fait de coureurs de niveaux proches. Tout se passe comme si chaque coureur filtrait, parmi l'ensemble des concurrents, ceux qui sont dignes d'intérêt pour lui et par rapport auxquels il lui importe de se situer, à savoir ceux qui sont d'un niveau proche du sien et susceptible de le menacer (Wouters, 1998). Cela dénote que les coureurs importent dans la situation la connaissance préalable qu'ils ont de leurs différents rivaux : c'est, par exemple, sur la foi des réalisations passées de Hicham El Guerrouj (alors meilleur du monde dans sa spécialité) qu’Ali estime cet athlète intouchable. Quand bien même la course à pied appartient à ces univers où les classements interindividuels se font et se défont dans et par l'interaction et ne peuvent perdurer quau prix d'un travail permanent d'entretien (Bourdieu, 1976), il y a une certaine inertie des classements antérieurs. Les résultats passés persistent sous forme de barrières intérieures (Elias, 1996 [1989]) : à mesure qu'il se confronte à ses adversaires, chaque athlète intériorise un sens de sa propre place dans la hiérarchie athlétique et ce qu'il lui sera possible de faire lors d'une confrontation ultérieure (Schotté, 2015).

Privé de repères extérieurs comme ceux fournis par le chronomètre, le coureur réagit en fonction des autres ou, plus précisément, en fonction de la façon dont il les perçoit, sur la base de sa connaissance de leurs performances passées et de ce qu'il observe en situation. Vu sous cet angle, le pouvoir en course résulte du différentiel entre la perception de soi et la perception des autres, comme le suggère ici cet échange avec Azzedine à propos d'un concurrent qu'il estime supérieur :

- «Lui, je ne pouvais pas le suivre. Il est trop fort. 
- Comment tu sais qu'il est trop fort?

-Bah je le connais! Je sais qu'il est fort.

- Parce que tu connais ses «perf»?

-Bah oui... mais même, tu vois qu'il est fort quand tu cours avec lui, tu le sens.

-Tu sens que tu peux pas rivaliser avec lui?

-Bah oui, il est... pfff... tu le vois quoi».

Plus que la sensation, nécessairement imprécise, de fatigue, c'est la place objective et subjective que le coureur occupe dans la hiérarchie athlétique qui détermine ce dont il se croit capable et ce qu'il fait durant l'épreuve. La mise en série des récits de courses recueillis tout au long de l'enquête atteste que quand un athlète est relégué et/ou se sent dominé, il subit le rythme imposé par ses rivaux. Et les évocations de décrochages ou d'envies d'abandonner sont invariablement reliées à des moments où l’athlète est dépassé par le cours des événements. Inversement, quand un coureur se sent en position de force, il manifeste une attitude conquérante et c'est lui qui imprime le rythme. Ces indications découlant de témoignages individuels sont corroborées par des objectivations chiffrées. Il est en effet possible, lorsque qu'un cross se déroule sur un circuit fermé et donne lieu à plusieurs tours, de chronométrer les temps réalisés par les athlètes lors de chaque boucle ${ }^{12}$. Et il apparaît alors que, dans la très large majorité des cas, le vainqueur de la course est celui qui maintient le même rythme d'un bout à l'autre de la course, tous les autres concurrents ne faisant que ralentir'3 .

12 II s'agit bien ici d'un artefact objectivant propre à la démarche de recherche: alors que je fréquente les labours depuis plus de 25 ans, je n'ai jamais vu le moindre athlète (ni le moindre entraîneur) chronométrer un cross. Si les temps réalisés et consignés par les organisateurs sont consultés attentivement par les coureurs après l'épreuve, c'est au titre des écarts qu'ils donnent à voir et jamais en tant qu'indicateurs directs de la performance.

13 Il y a certes le cas de ceux qui reviennent de l'arrière: leur remontée dans le classement signifie qu'ils ont été incapables de suivre le rythme imposé en début de course. Mais, là encore, l'expérience apprend qu'un cross n'est jamais remporté par un coureur qui n'était pas aux avant-postes dès le début de course. 
Ces éléments étayent que le vainqueur est celui qui impose son allure aux autres qui lui abandonnent le commandement de leur effort. Cela amène à relier pouvoir sur les autres et maîtrise de son propre engagement: les athlètes ne dirigent pleinement leur effort que lorsqu'ils sont en situation de peser sur celui de leurs adversaires. Mettre ainsi en évidence, à la suite de Whyte (2002 [1943]) à propos des parties de bowling auxquelles il participa avec ses enquêtés, l'existence d'une dialectique entre la performance et la position - relation dialectique qui opère par la médiation de la manière dont l'individu se perçoit dans la situation et des émotions qui en découlent ${ }^{14}$ - conduit nécessairement à inclure les effets de la seconde dans la compréhension de la première. Ce qui est ici entendu par «position» se décline de deux façons : d’abord, la position occupée aux différents moments de la course (étant entendu que cette position est susceptible de fluctuations au cours d'une même épreuve) ; ensuite, la position occupée avant la course. Les concurrents n’arrivent en effet pas sur la ligne de départ uniquement pourvus de leurs compétences techniques. Ils y importent l'ensemble de leur histoire personnelle (qui détermine notamment la signification que revêt pour eux le verdict compétitif) et de leurs performances passées, ces dernières étant le plus souvent connues de leurs rivaux.

L’importance des performances antérieures et des hiérarchies interindividuelles qu'elles contribuent à établir se repère dans le cas évoqué ci-dessous. Il se rapporte à un athlète qui dominait sa spécialité sur le plan mondial et qui, le soir d'une course où il fut, de façon exceptionnelle, menacé par un adversaire jusqu'aux derniers mètres de l'épreuve, m'expliqua qu'il avait maîtrisé la situation de bout en bout :

-C'était serré dans la dernière ligne droite!

-Ouais, je sentais qu'il était juste à côté. Mais je n’ai pas paniqué. Je savais que j'étais plus fort que lui.

-Mais il était tout prêt! Vous n’avez pas eu peur que... 
- Non je contrôlais. Je sentais que... Et dans le dernier virage je l'ai laissé croire. Comme ça il s’est décalé. Et quand il était à l'extérieur [dans le virage, ce qui signifie que l'athlète couvre une distance légèrement plus grande que celui qui reste à la corde], j’ai accéléré.

La sécurité procurée par le sentiment de sa supériorité - maintes fois répétées lors de confrontations antérieures - permet à cet athlète de se soustraire de la pression du présent et de mettre à distance la menace que représentait son adversaire. Bien qu'en situation de danger objectif, il ne s'est jamais senti en péril. Est ainsi mis en évidence le lien entre maîtrise des autres et maîtrise de soi : un coureur contrôle son engagement quand il est en mesure d'imposer son allure, et, inversement, il subit le rythme quand il est dominé par ses rivaux.

Tout ceci prouve que les possibles d'un athlète ne sont pas réductibles à la seule mise en jeu de ses qualités physiques, mêmes conçues comme travaillées en amont par le social. Ce dont un coureur s'avère capable est aussi fonction de ce qui se joue en situation, pendant la compétition qui est une interaction de nature particulière. Est ainsi restituée toute l'épaisseur sociale dont est chargée la performance en course à pied. Loin d'être réductible à des lois biologiques, cette dernière procède bien d'un ensemble de conditions sociales qui déterminent les possibles d'un athlète.

\section{CONCLUSION}

Le cas étudié invite à considérer le biologique non pas comme le contraire du social mais comme le support de la vie sociale (pour exister socialement, il vaut mieux, à de rares exceptions près ${ }^{15}$, être vivant que mort). Suivant cette perspective, le travail du sociologue ne consiste pas à s'opposer au biologique mais à rendre compte de ce que le social fait à des potentialités physiologiques dont il n'y a pas lieu de penser qu'elles puissent être, à elles seules, au principe de différences interindividuelles socialement efficientes ${ }^{16}$. Il ne

I5 Je fais ici référence au cas de la «vie posthume», lorsque les réalisations d'un individu continuent de le faire vivre symboliquement après sa mort.

I6 Si le fait d'être grand - propriété biologique innée - constitue un atout dans certaines pratiques, c'est que celles-ci ont été façonnées de telle façon qu'elles favorisent certaines 
s'agit donc pas de réduire le social au statut d'environnement qui offrirait des occasions d'actualisation à des différences déjà-là et irréductibles à l'analyse sociologique (Schotté, 20I3) mais au contraire de l'envisager comme le lieu de détermination des possibles. L’apport des sciences sociales réside alors dans la possibilités qu'elles offrent de mettre au jour tout ce qui conduit à l'existence de différences entre les individus. Et s'«il n’y a jamais lieu d'être certain du caractère naturel des inégalités que l'on constate dans une situation sociale donnée $[. .$.$] tant qu'on n’a pas exploré toutes les voies par où agissent les$ facteurs sociaux d'inégalités » (Bourdieu et Passeron, 1964, p. I03), il ne faut pas pour autant assimiler le naturel au biologique. Il importe en effet de ne pas confondre ce qui est décrit comme découlant de la nature et ce qui relève du biologique $^{17}$. Si le premier registre renvoie à un ordre immuable qui s'impose de lui-même, le second relève d'une dimension très malléable, ouverte aux déterminations sociales. Il convient donc de prendre en compte la façon dont le social sarticule au biologique pour mettre au jour ce que le premier fait aux potentialités offertes par le second. Dans ces conditions, la meilleure arme à opposer au biologisme ${ }^{18}$ (qui décrit les comportements comme découlant des lois d'un ordre immuable) n'est assurément pas celle du sociologisme mais celle d'un constructivisme qui prend acte de la matérialité des corps et de la façon dont le social les affecte et les transforme, de façon différente suivant les conditions dans lesquelles ils évoluent.

caractéristiques physiques. Mais la taille ne peut à elle seule expliquer la performance en basket-ball par exemple: nombreux sont les cas de joueurs recrutés sur la base de leur très grande taille et ayant eu des carrières limitées.

17 Voir à ce propos le travail de Meloni (20l4b) qui montre que l'association qui conduit à assimiler le biologique au naturel est historiquement datée, insoutenable sur le plan logique et aujourd'hui rejetée par une grande partie des biologistes eux-mêmes.

I8 Le raisonnement se cantonne ici à des considérations strictement intellectuelles. II ne faut toutefois pas perdre de vue que, comme la phrase d'Elias placée en exergue de ce texte le rappelle, les oppositions disciplinaires sont aussi des concurrences institutionnelles. L'impérialisme des sciences biologiques ne procède peut-être pas tant d'un rejet du social que d'une façon particulière de l'envisager et d'une volonté d'imposer leur modèle scientifique (régime épistémologique, mode de publication, etc.) aux sciences sociales. 


\section{BIBLIOGRAPHIE}

BECKER Howard, 1985 (1963), Outsiders. Études de sociologie de la déviance, Paris, Métailié.

BENDELOW Gillian A. et WILLIAMS Simon J., 1995, «Transcending the Dualisms: Towards a Sociology of Pain», Sociology of Health and IIIness, 17-2, p. 139-165.

BESNIER Niko, 2012, «The Athlete's Body and the Global Condition: Tongan Rugby Players in Japan», American Ethnologist, 39-3, p.491-5I0.

BOLTANSKI Luc, 197I, «Les usages sociaux du corps», Annales ESC, 26-I, p. 205-233.

BOURDIEU Pierre, 1976, «Les modes de domination», Actes de la recherche en sciences sociales, 2-3, p. I22-132.

-, 1997, Méditations pascaliennes, Paris, Éditions du Seuil.

- et PASSERON Jean-Claude, 1964, Les Héritiers. Les étudiants et la culture, Paris, Éditions de Minuit, «Le sens commun».

BRUANT Gérard, 1992, Anthropologie du geste sportif. La construction sociale de la course à pied, Paris, PUF.

COLLINSON Jacquelyn A., 2008, «Running the Routes Together. Corunning and Knowledge in Action », Journal of Contemporary Ethnography, 37-I, p. 38-6I.

DÉTREZ Christine, 2002, La Construction sociale du corps, Paris, Éditions du Seuil.

ELIAS Norbert, 1973 (1939), La Civilisation des moeurs, Paris, Calmann-Levy.

-, 1996 (1989), The Germans: Power Struggles and the Development of Habitus in the Nineteenth and Twentieth Centuries, Cambridge, Polity Press.

-, 2015 (1991), La Théorie des symboles, Paris, Éditions du Seuil.

GOFFMAN Erving, 1970, Strategic Interaction, Oxford, Basil Blackwell.

HOCHSCHILD Arlie R., 1979, «Emotion Work, Feeling Rules, and Social Structure», American Journal of Sociology, 85-3, p.55I-575.

KATZ Jack, 1999, How Emotions Work, Chicago, The University of Chicago Press.

- et CSORDAS Thomas J., 2003, «Phenomenological Ethnography in Sociology and Anthropology», Ethnography, 4-3, p. 275-288. 
LIGNIER Wilfried, 2015, «La nature humaine nous laisse tous indistincts», Genèses, 100/101-3/4, p. 162-168.

MAUSS Marcel, 1978 (1934), «Les techniques du corps», in Id., Sociologie et Anthropologie, Paris, PUF, p. 363-386.

MELONI Maurizio, 2014a, «How Biology Became Social, and What It Means for Social Theory», The Sociological Review, 62-3, p. 593-614.

-, 20I4b, «Biology without Biologism: Social Theory in a Postgenomic Age», Sociology, 48-4, p. 731-746.

NOAKES Timothy D., 2012, «Fatigue is a Brain-Derived Emotion that Regulates the Exercise Behavior to Ensure the Protection of Whole Body Homeostasis », Frontiers in Physiology, 3-82, [en ligne]: <doi: 10.3389/fphys.20 I2.00082>.

SCHOTTÉ Manuel, 2012, La Construction du «talent». Sociologie de la domination des coureurs marocains, Paris, Raisons d'Agir.

-, 2013, «Le don, le génie et le talent. Critique de l'approche de Pierre-Michel Menger», Genèses, 93-4, p. 144-164.

-, 2015, «Dans la course. La construction d'une hiérarchie en action», Actes de la recherche en sciences sociales, 209, p. 100-115.

SCHUTZ Alfred, 195I, «Making music together: a study in social relationship», Social Research, 18, p.76-97.

VANDEBROECK Dieter, 2015, «Distinctions charnelles. Obésité, corps de classe et violence symbolique», Actes de la recherche en sciences sociales, 208, p. 14-39.

WACQUANT Loïc, 1995, «Pugs at Work: Bodily Capital and Bodily Labor Among Professional Boxers», Body \& Society, I-I, p. 65-94.

-, 2000, Corps et âme. Carnets ethnographiques d'un apprenti boxeur, Marseille, Agone, «Mémoires sociales».

WHYTE William F., 2002 (1943), Street Corner Society. La structure sociale d'un quartier italo-américain, Paris, La Découverte.

WILMORE Jack H. et COSTILL David L., 1994, Physiology of Sport and Exercise, Champaign, Human Kinetics.

WOUTERS Cas, 1998, «How Strange to Ourselves are our Feelings of Superiority and Inferiority», Theory, Culture and Society, I5-I, p. I3I-150. 